\title{
Correlation analysis of epicardial adipose tissue volume quantified by computed tomography images and coronary heart disease under optimized reconstruction algorithm
}

\author{
Zhenwei Miao', Hongyan Yang², Bofen Liü, Wengui Li ${ }^{4}$
}

\begin{abstract}
Objectives: This paper was aimed to explore the adoption value of low-dose computed tomography (CT) imaging based on optimized ordered subset expectation maximization (OSEM) reconstruction algorithm in the correlation analysis between epicardial adipose tissue (EAT) volume and coronary heart disease (CHD). Methods: A total of 110 patients with CHD were selected for CT angiography (CTA) and coronary arteriography (CAG) examinations from October 2017 to October 2019. The predictive value of EAT for CHD was analyzed via receiver operating characteristic (ROC) curve.

Results: The results showed that the iteration time and error of the improved OSEM reconstruction algorithm were better than that of MLEM algorithm under the same number of iterations. Age, smoking, hypertension, diabetes, and EAT in control group were obviously lower in contrast to those in CHD group $(\mathrm{P}<0.05)$. EAT in control group was $(124.50 \pm 26.72) \mathrm{mL}$, and EAT in the CHD group was $(159.41 \pm 38.51) \mathrm{mL}$. EAT $(B=0.023, P=0.003)$ was an independent risk factor for $C H D$, which was suggested by Multiple linear regression analysis. Moreover, EAT was a risk factor for $\mathrm{CHD}$, and was positively correlated with the degree and NSCV.

Conclusion: The optimized OSEM algorithm was used to improve the reconstruction quality of low-dose CT images and used in quantitative measurement of epicardial fat volume. Results showed EAT was an independent risk factor for CHD, and was positively correlated with the number of coronary lesions and Gensini score. It was of great value for the prediction of CHD.
\end{abstract}

KEYWORDS: Ordered subset expectation maximization reconstruction algorithm; Coronary heart disease; Computed tomography; Epicardial adipose tissue; Gensini scoring.

doi: https://doi.org/10.12669/pjms.37.6-WIT.4882

How to cite this:

Miao Z, Yang H, Liu B, Li W. Correlation analysis of epicardial adipose tissue volume quantified by computed tomography images and coronary heart disease under optimized reconstruction algorithm. Pak J Med Sci. 2021;37(6):1677-1681. doi: https://doi.org/10.12669/pjms.37.6-WIT.4882

This is an Open Access article distributed under the terms of the Creative Commons Attribution License (http://creativecommons.org/licenses/by/3.0), which permits unrestricted use, distribution, and reproduction in any medium, provided the original work is properly cited.

1. Zhenwei Miao, Master of Medicine.

Department of Radiology,

2. Hongyan Yang, Bachelor's Degrees.

Department of Nursing,

3. Bofen Liu, Bachelor's Degrees.

Department of Nursing,

4. Wengui Li, Bachelor's Degrees.

Department of Radiology,

1-4: Tianjin Baodi Hospital, Tianjin City 301800, China.

Correspondence:

Wengui Li,

Department of Nursing, Tianjin Baodi Hospital,

Tianjin City 301800, China.

E-mail: liwenguitjbdh@163.com

* Received for Publication:

* Revision Received:

* Final Revision Accepted:
April 5, 2021

June 12, 2021

July 5, 2021

\section{INTRODUCTION}

Many studies have revealed that EAT is closely related to the occurrence of coronary atherosclerosis, and may be a new risk factor for CHD..$^{1-3}$ The measurements of EAT include ultrasound, ${ }^{4-6} \mathrm{MRI}^{7}$ and CT. ${ }^{8} \mathrm{CT}$ imaging technology is currently the preferred method for measuring EAT in clinics, and it can achieve accurate quantitative measurement of the total volume of EAT. Therefore, using CT technology to explore the volume changes of the EAT in CHD patients is of great significance for the early prevention of $\mathrm{CHD}$ and the subsequent effective treatment. 
Therefore, the optimized OSEM algorithm was applied in low-dose CT image reconstruction for CHD patients to obtain high-quality images. Quantitative measuring of the volume of epicardial fat in patients with CHD was performed, and its correlation with the incidence of CHD was further explored, to provide accurate imaging basis for early diagnosis of CHD.

\section{METHODS}

One hundred and ten patients with CHD who were examined by CTA and CAG in our hospital from October 2017 to October 2019 were selected. Patients with CAG stenosis no less than $50 \%$ were classified as CHD group, and the rest as control group. Among them, there were 42 patients (28 males) in control group with an average age of $59.85 \pm 7.67$ years. There were 68 patients (42 males) in the CHD group with an average age of $61.67 \pm 6.28$ years.

Inclusion criteria: I, patients were 30-84 years old; II, patients received CTA and CAG joint inspection; III, the image quality was favorable.

Exclusion criteria: I, patients with coronary artery bypass graft; II, patients with coronary artery stenting; III, patients with congenital heart disease; IV, poor image quality; $\mathrm{V}$, patients with atrial fibrillation and arrhythmia; VI, patients with cardiomyopathy or valvular disease. This experiment had been approved by the ethics committee of the hospital, and the patients involved in the study had known and agreed with it.

ETA volumetric measurement: Two experienced physicians were selected to conduct ETA measurement using Vitrea post-processing workstation related software, and the results were averaged. The fat threshold range was set from 150 to $-50 \mathrm{Hu}$. Firstly, the fat tissue was extracted, mainly from the myocardium and the visceral pericardium. Then, measurements were made from the beginning of the left pulmonary artery to the apex of the left ventricle.

The detection was completed by two senior interventional cardiologists. Left and right coronary arteriography were performed by conventional body position imaging, and heparin was used for anticoagulation during the operation. Coronary angiography referred to diagnosing and analyzing the location, severity, and NSCV of coronary artery stenosis. The left main coronary artery was classified as two-vessel lesion, while other vascular lesions were classified into single-vessel, double-vessel, and multiple-vessel lesions according to the NSCV.

The Gensini scoring system was used to evaluate the degree of vascular lesions. ${ }^{9}$ The stenosis coefficient value was determined, the diameter of blood vessel was " $0-25 \%, 51 \%-75 \%$, $76 \%-90 \%, 91-99 \%$, and $100 \%$ ", which counted 1, 2, $4,8,16$, and 32 points, respectively. The lesion site coefficient value was determined. The coefficient of left main coronary artery was 5 . The coefficients of the proximal left anterior descending and the proximal left rotatory branch were both 2.5 . The coefficient of the middle part of left anterior descending branch was 1.5. The coefficients of the distal left anterior descending branch, the distal left anterior branch, the obtuse margin branch, the right coronary artery (proximal, middle, and distal), and the posterior descending branch were all 1 . The coefficient of the second diagonal branch was 0.5 . The value of lesion site coefficient $\times$ stenosis coefficient value was the total score.

OSEM is an accelerated iterative algorithm. The main principle is sorting the projection data first, and then dividing it into $L$ subsets $\left\{S_{1}, S_{2}, \mathrm{~S}_{3}, \cdots, \mathrm{S}_{L}\right\}$. In each sub-iteration process, all projection data of a certain subset are used to correct the image. ${ }^{10}$ Therefore, the image in each iteration is updated L times, which makes the convergence rate increase rapidly and the CT image reconstruction time becomes shorter. In addition, OSEM algorithm also has good antinoise ability and high spatial resolution. The iteration equation is as follows.

In the above equation, $j$ is the pixel; $i$ is the detector number; qij is the probability; $\mathrm{k}$ is the number of iterations; gkj is the image after $\mathrm{k}$ iterations; yi is the observation value; sm is the $\mathrm{m}$-th subset; and $1 \leq \mathrm{m} \leq \mathrm{L}$.

The specific implementation of the OSEM algorithm is divided into steps such as initial value, subset projection of the probability matrix, residual calculation, correction factor calculation, image correction, and loop iteration. ${ }^{11}$ The calculation of the initial value is as follows.

$$
\mathrm{g}^{0}=0
$$

Projection of probability matrix on subset is as follows.

$$
\tilde{q}_{i}=\sum_{j=1}^{N} p_{i j} g_{i}, i \in S_{m}
$$

The ratio of the actual projection qi to the theoretical projection is the residual. 
Zhenwei Miao et al.

Table-I: Risk factors for CHD.

\begin{tabular}{|c|c|c|c|c|c|c|c|}
\hline \multirow{2}{*}{ Factor } & \multirow{2}{*}{ Wals } & \multirow{2}{*}{$B$} & \multirow{2}{*}{$S E$} & \multirow{2}{*}{$O R$} & \multicolumn{2}{|c|}{$95 \% C I$} & \multirow{2}{*}{$P$} \\
\hline & & & & & Lower limit & Upper limit & \\
\hline Constant & 0.005 & 0.167 & 2.654 & 1.176 & - & - & 0.864 \\
\hline EAT & 8.365 & 0.023 & 0.006 & 1.017 & 1.003 & 1.038 & 0.003 \\
\hline Uric acid & 0.768 & 0.007 & 0.018 & 0.996 & 0.975 & 1.001 & 0.354 \\
\hline Age & 0.685 & 0.236 & 0.028 & 0.398 & 0.956 & 1.023 & 1.007 \\
\hline BMI & 2.187 & -0.114 & 0.073 & 0.142 & 0.745 & 1.037 & 0.879 \\
\hline Gender & 0.032 & -0.117 & 0.627 & 0.874 & 0.238 & 3.109 & 0.865 \\
\hline Smoking history & 3.218 & -1.105 & 0.542 & 0.321 & 0.087 & 1.006 & 0.718 \\
\hline Hypertension & 3.265 & -1.486 & 0.821 & 0.068 & 0.039 & 1.076 & 1.206 \\
\hline Diabetes & 2.483 & -0.802 & 0.486 & 0.102 & 0.154 & 1.208 & 0.432 \\
\hline
\end{tabular}

$$
\Delta_{i}=\frac{q_{i}}{\tilde{q}_{i}}
$$

Correction factor calculation is as follows.

$$
C_{i}=\frac{1}{\sum_{i \in S_{m}} p_{i j}} \sum_{i \in S_{m}} p_{i j} \Delta_{i}
$$

$\mathrm{CT}$ reconstruction image correction is as follows.

$$
g_{j}^{k+1}=g_{j}^{k} * C_{i}
$$

Optimizing of the partitioning of OSEM algorithm subsets is as follows. I, the number of projections was fully considered, so that all subsets obtained by the division have uniform projection angles, and the subsets should be balanced and symmetrical; II, in the subset sorting process, it should be ensured that the distance between the projection directions of the adjacent two subsets is the largest.

\section{RESULTS}

The simulation data was a $256 \times 256$ sheepLogan phantom, and each subset in the OSEM

Table-II: Correlation of EAT with Gensini score and the NSCV.

\begin{tabular}{ccc}
\hline & Gensisi score & $\begin{array}{c}\text { Number of stenosed } \\
\text { coronary vessel }\end{array}$ \\
\hline $\mathrm{r}$ & 0.327 & 0.416 \\
$\mathrm{P}$ & 0.002 & 0.000 \\
\hline
\end{tabular}

algorithm was set with 6 projection directions. When the projection interval was $1^{\circ}$, the iteration time was 223.56, and the error was 0.0465. The accuracy of the reconstructed image was very high. When the number of iterations increased, the error decreased and the imaging quality would be higher. The iteration time increased with the number of iterations.

The projection interval was set as $1^{\circ}$ and the number of projection directions as 6 . When the number of iterations was the same, the OSEM algorithm had shorter iteration time and smaller error. When the MLEM algorithm was iterated for 30 times, the error was at the same level as the OSEM algorithm after 1 iteration. It can be concluded that the calculation speed of OSEM algorithm was higher in contrast to that of MLEM algorithm, the reconstruction time was greatly shortened, and the imaging quality was better.

The results showed that middle part of anterior descending branch was about $80 \%$, the proximal stenosis of the circumflex branch was between $70 \%$ and $75 \%$, and the Gensini score was 36 . With CHD as the dependent variable and gender, BMI, uric acid, smoking, hypertension, diabetes, and EAT as the independent variables for Logistic multiple regression, EAT $(B=0.023, \mathrm{P}=0.003)$ was an independent risk factor for CHD (Table-I).

Spearman correlation analysis suggested that EAT volume was positively correlated with Gensini score ( $\mathrm{r}=0.327, \mathrm{P}=0.002)$; EAT volume was positively correlated with the NSCV $(r=0.416$, $\mathrm{P}=0.000$ ) (Table-II). 
Among CHD patients, there were 10, 19, and 19 cases of single-vessel, double-vessel, and multivessel disease (Fig.1A). The Gensini score of double-vessel disease was notably higher in contrast to that of single-vessel disease, $\mathrm{P}<0.05$; the Gensini score of multivessel disease was extremely notably higher in contrast to those of double-vessel disease and single-vessel disease, $\mathrm{P}<0.01$ (Fig.1B). The EAT value of multivessel disease was extremely notably higher in contrast to those of double-vessel disease and single-vessel disease, $\mathrm{P}<0.01$ (Fig.1C).

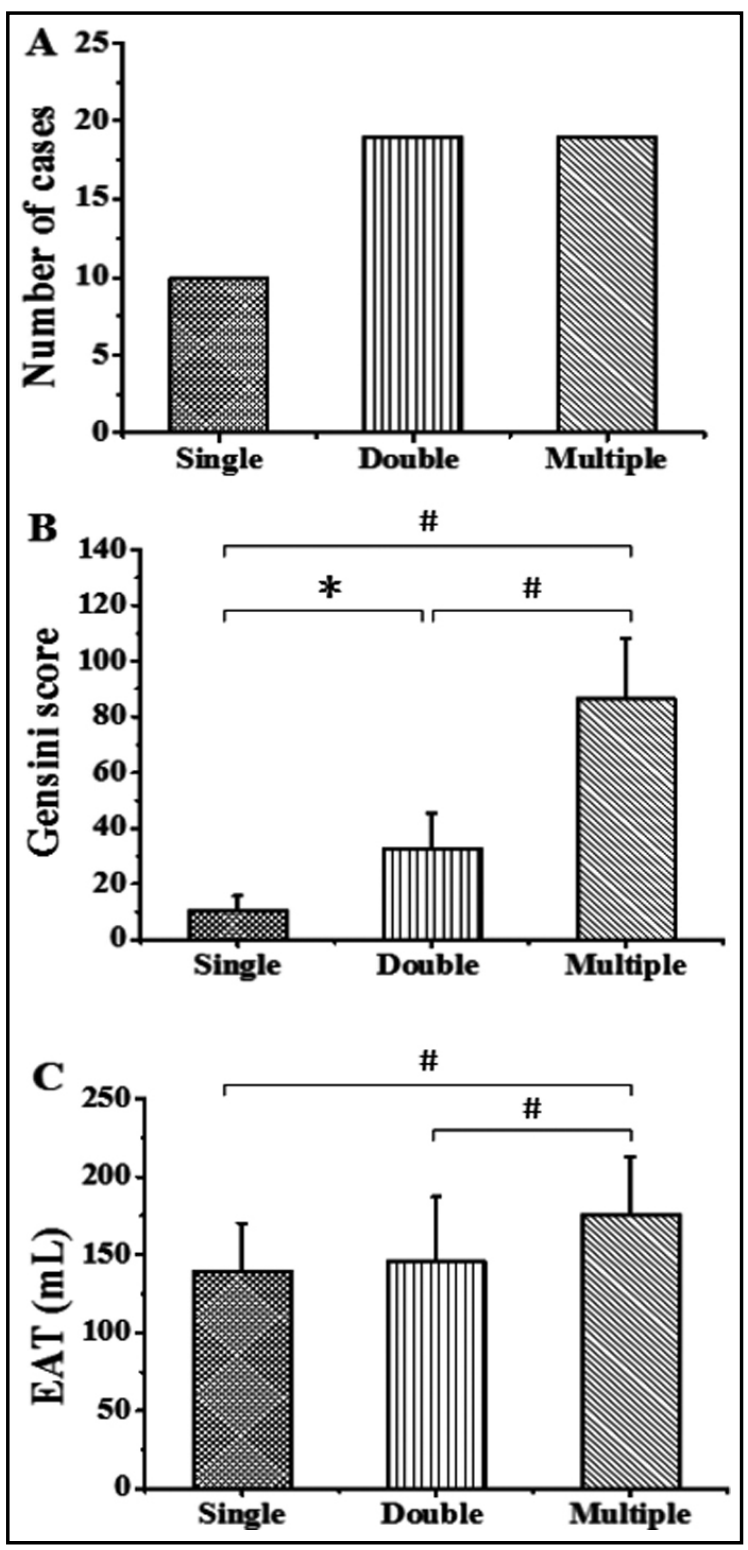

Fig.1: The relationship between

NSCV and EAT and Gensini.

(A: number of patients; B: Gensini score;

$\mathrm{C}$ : EAT; * meant $\mathrm{P}<0.05$; \# meant $\mathrm{P}<0.01$.)

\section{DISCUSSION}

With the rapid development of medical imaging technology, X-ray, MRI, and CT have been widely used in the disease diagnosis, treatment, and efficacy evaluation. CT imaging is often used in the examination of chest and abdomen diseases. Chest CT examination has the advantages of clear structure, but the image is not very clear for soft tissue, and the radiation amount of $\mathrm{CT}$ imaging is much higher than that of X-ray. ${ }^{12,13}$ In order to reduce the amount of radiation to the human body during CT examination, low-dose CT is often used for clinical examination. However, as the intensity of CT scan decreases, the quality of the reconstructed image will be greatly reduced. ${ }^{14}$ Therefore, the reconstruction of low-dose CT images has become the focus of attention, and the use of iterative reconstruction algorithms to reconstruct low-dose CT images can obviously solve the reduced signal-to-noise ratio. ${ }^{15}$ The OSEM algorithm can divide the projection data into $\mathrm{N}$ different subsets through an iterative reconstruction process, and each subset is iterated. ${ }^{16,17}$ In this study, OSEM algorithm was used to reconstruct low-dose CT images. The results showed that, compared with MLEM algorithm, OSEM algorithm can significantly shorten the iteration time, and the image error after reconstruction was smaller. The MLEM algorithm has shortcomings, such as slow iteration speed, which is consistent with the results of this study. ${ }^{18}$

\section{CONCLUSIONS}

The optimized OSEM algorithm was used to improve the reconstruction quality of lowdose CT images and used in quantitative measurement of epicardial fat volume. Results showed EAT was an independent risk factor for CHD, and was positively correlated with the number of coronary lesions and Gensini score. It was of great value for the prediction of CHD.

\section{REFERENCES}

1. Wirtz PH, von Kanel R. Psychological Stress, Inflammation, and Coronary Heart Disease. Curr Cardiol Rep. 2017;19(11):111. doi: 10.1007/s11886-017-0919-x

2. Gordon T, Castelli WP, Hjortland MC, Kannel WB Dawber TR. High density lipoprotein as a protective factor against coronary heart disease. The Framingham Study. Am J Med. 1977;62(5):707-714. doi: 10.1016/00029343(77)90874-9 
3. Xiao J, Lu Y, Yang X. Ultrasound Detection of Epicardial Adipose Tissue Combined With Ischemic Modified Albumin in the Diagnosis of Coronary Heart Disease. Heart Surg Forum. 2020;23(4):E461-E464. doi: 10.1532/ hsf.3015

4. Patel VB, Shah S, Verma S, Oudit GY. Epicardial adipose tissue as a metabolic transducer: role in heart failure and coronary artery disease. Heart Fail Rev. 2017;22(6):889-902. doi: 10.1007/s10741-017-9644-1

5. Yuan M, Wu H, Li R, Yu L, Zhang J. Epicardial adipose tissue characteristics and CT high-risk plaque features: correlation with coronary thin-cap fibroatheroma determined by intravascular ultrasound. Int J Cardiovasc Imaging. 2020;36(11):2281-2289. doi: 10.1007/s10554-02001917-2

6. Haines DE, Wright M, Harks E, Deladi S, Fokkenrood S, et al. Near-Field Ultrasound Imaging During Radiofrequency Catheter Ablation: Tissue Thickness and Epicardial Wall Visualization and Assessment of Radiofrequency Ablation Lesion Formation and Depth. Circ Arrhythm Electrophysiol. 2017; 10(12): e005295. doi: 10.1161/ CIRCEP.117.005295

7. Leo LA, Paiocchi VL, Schlossbauer SA, Ho SY, Faletra FF. The Intrusive Nature of Epicardial Adipose Tissue as Revealed by Cardiac Magnetic Resonance. J Cardiovasc Echogr. 2019;29(2):45-51. doi: 10.4103/jcecho.jcecho_22_19

8. Aliyari Ghasabeh M, Te Riele A, James CA, Chen $H$, Tichnell C, et al. Epicardial Fat Distribution Assessed with Cardiac CT in Arrhythmogenic Right Ventricular Dysplasia/Cardiomyopathy. Radiology. 2018;289(3):641648. doi: 10.1148 /radiol.2018180224

9. Wang $\mathrm{P}$, Qiang $\mathrm{H}$, Song $\mathrm{Y}$, Dang $\mathrm{Y}$, Luan $\mathrm{H}$, et al Association between Nonalcoholic Fatty Liver and Gensini Score in Patients with Coronary Heart Disease: A CrossSectional Study. Cardiology. 2019;144(3-4):90-96. doi: 10.1159/000502111

10. Kortelainen MJ, Koivumaki TM, Vauhkonen MJ, Hakulinen MA. Time-modified OSEM algorithm for more robust assessment of left ventricular dyssynchrony with phase analysis in ECG-gated myocardial perfusion SPECT. EJNMMI Phys. 2019;6(1):30. doi: 10.1186/s40658-0190261-z

11. Aijing $\mathrm{H}$, Xianguo $\mathrm{T}$, Rui S, Honglong Z. An improved OSEM iterative reconstruction algorithm for transmission tomographic gamma scanning. Appl Radiat Isot. 2018;142:51-55. doi: 10.1016/j.apradiso.2018.09.001
12. Sarikaya I, Elgazzar AH, Sarikaya A, Alfeeli M. Normal bone and soft tissue distribution of fluorine-18-sodium fluoride and artifacts on 18F-NaF PET/CT bone scan: a pictorial review. Nucl Med Commun. 2017;38(10):810-819. doi: 10.1097/MNM.0000000000000720

13. Ren L, Rajendran K, McCollough CH, Yu L. Radiation dose efficiency of multi-energy photon-counting-detector CT for dual-contrast imaging. Phys Med Biol. 2019;64(24):245003. doi: 10.1088/1361-6560/ab55bf

14. Moen TR, Chen B, Holmes DR, Duan X, Yu Z, et al Low-dose CT image and projection dataset. Med Phys. 2021;48(2):902-911. doi: 10.1002/mp.14594

15. Wolterink JM, Leiner T, Viergever MA, Isgum I. Generative Adversarial Networks for Noise Reduction in Low-Dose CT. IEEE Trans Med Imaging. 2017;36(12):2536-2545. doi: 10.1109/TMI.2017.2708987

16. van Nierop BJ, Prince JF, van Rooij R, van den Bosch $M$, Lam M, et al. Accuracy of SPECT/CT-based lung dose calculation for Holmium-166 hepatic radioembolization before OSEM convergence. Med Phys. 2018;45(8):38713879. doi: $10.1002 / \mathrm{mp} .13024$

17. Razifar $P$, Sandstrom $M$, Schnieder $H$, Langstrom B, Maripuu E, et al. Noise correlation in PET, CT, SPECT and PET/CT data evaluated using autocorrelation function: A phantom study on data, reconstructed using FBP and OSEM. BMC Med Imaging. 2005;5:5. doi: 10.1186/14712342-5-5

18. Dey J, King MA. Theoretical and Numerical Study of MLEM and OSEM Reconstruction Algorithms for Motion Correction in Emission Tomography. IEEE Trans Nucl Sci. 2009;56(5):2739-2749. doi: 10.1109/tns.2009.2021765

\section{Authors Contribution:}

ZM: Conceived the study, literature review, data analysis, preparing the draft of paper.

HY \& BL: Helped in design, data collection drafting \& critical revision.

WL: Takes the responsibility and is accountable for all aspects of the work in ensuring that questions related to the accuracy or integrity of any part of the work are appropriately investigated and resolved. 